\title{
Study of Phishing Attacks and Preventions
}

\author{
Tenzin Dakpa \\ Department of Computer Science, \\ Christ University, \\ Karnataka, Bangalore
}

\author{
Peter Augustine \\ Department of Computer Science, \\ Christ University, \\ Karnataka, Bangalore
}

\begin{abstract}
The Internet is a great discovery for ordinary citizens correspondence. People with criminal personality have found a method for taking individual data without really meeting them and with minimal danger of being gotten. It is called Phishing. Phishing represents a huge threat to the web based business industry. Not just does it smash the certainty of clients towards online business, additionally causes electronic administration suppliers colossal financial misfortune. Subsequently, it is fundamental to think about phishing. This paper gives mindfulness about Phishing assaults and hostile to phishing apparatuses.
\end{abstract}

\section{General Terms}

DNS, Spear phishing, gaming, antivirus, scam and spam.

\section{Keywords}

Phishing, Phishing Types, Prevention of Phishing, Antiphishing tool.

\section{INTRODUCTION}

Phishing is the source of getting sensitive data, for example, usernames, passwords, and charge card points of interest (through various ways to get cash), frequently for malignant reasons, by taking on the appearance of a strong element in an electronic correspondence. The word used is a new term as a homophone as similarity with activity taken place. Interchanges indicating to be from mainstream social sites, sell off locales, banks, online installment processors. Phishing leads us to virus contaminated link sites which in turn confuses to the users.. Phishing is regularly done by email spoofing or texting, and it frequently guides user to enter points of interest at a fake site whose look and feel same as the original one. Attempts to handle the increasing number of phishing should be met by preparing the clients bringing in awareness and other efforts to establish protection various anti-phishing tools. Numerous sites have now made optional instruments for applications, similar to maps for redirection. However clients ought not to utilize similar passwords anyplace on the web[1].

\section{TYPES OF PHISHING}

\subsection{Clone Phishing}

The kind of phishing assault by which a genuine and beforehand conveyed, an email hold within a connection or connection has had its substance and it used to make a practically indistinguishable or cloned email. The connection or connection inside the email supplanted with a malevolent form and after that sent from an email delivery mock to seem to originate from the first user. It might sate to resend of the first or a redesigned to the first. This system could be utilized to turn (by implication) from a formerly tainted machine and pick up a toehold on different computers, by abusing the faith in people connected with the induced association because of both sides accepting the first email[6].

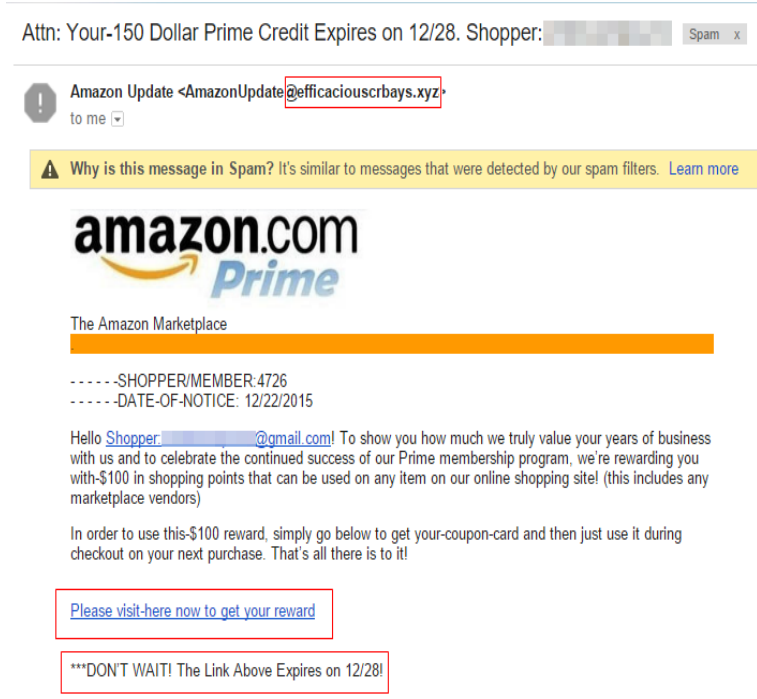

Fig 1: Clone Phishing

\subsection{Spear Phishing}

Here the malware, virus is targeted at a particular group of people or organizations have been named skewer phishing. Attackers collect entire information relating to their objective of accomplishment. This system is ranked the top on internet, having $91 \%$ of assaults.

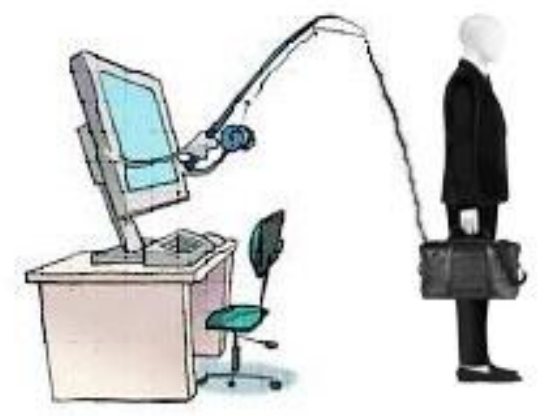

Fig 2: Spear Phishing

\subsection{Social Networking on Mobile}

Because of the ascent in the quantity of clients getting to the Internet through advanced cells, long range, interpersonal communication sites have extended their administrations on PDAs, including informing, talking, photograph seeing, and so forth. This expansion in clients has opened more ways to aggressors because there are currently more potential casualties. Henceforth, assailants have made phishing sites on long range interpersonal communication brands guaranteeing to give these administrations on advanced cells. 


\subsection{Gaming}

Gaming has turned into an inexorably prevalent part of interpersonal interaction. Symantec assessed gaming and found that it included 13 percent of the focused on applications. Gaming applications in person to person communication generally require different sorts of credit focuses to advance to larger amounts of the amusement. Some of these credit focuses normally require online installment. The phishing sites trap clients by giving fake offers of free credit focuses on these gaming applications.

\subsection{DNS Base Phishing}

Pharming is an assault meaning to divert a site movement to another malicious site. Harming meddle with the determination of space name service return an IP address, so area name of the bona fide site mapped onto IP address of rebel website.DNS phishing is delineated.

On the off chance that we are writing the area name www.icicbank.com in the delivery bar, it is diverted to www.google.com.

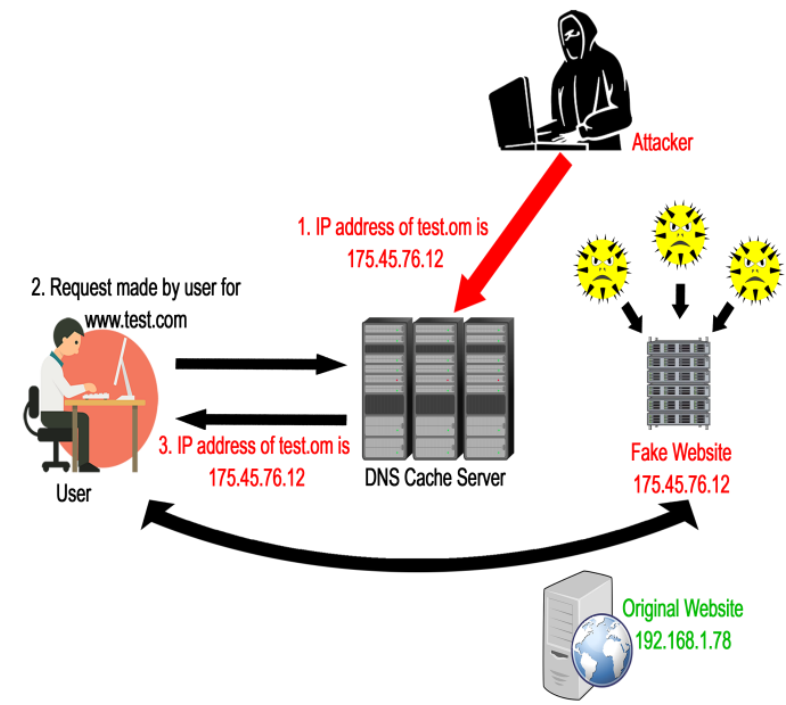

Fig 3: DNS Based Phishing

\subsection{Live Chat}

Live chat is a new twist to phishing attack where victim are tricked into getting more information via live chat..This type of phishing mainly occurs in online banking website where victim add fake live chat support window to their online banking sites to make them more real and reveal all the sensitive information from victim. Likewise the phishing assaults indicate fake offers of free sex talk to bait end clients into entering their login certifications

\subsection{Whaling}

Certain phishing skills are set to target specially superior administrators, organizations, and the name whaling was instituted for such types of assaults. On account of this type of phishing, the disguising site is of a higher official class frame and representing particular client of organization. The content of this phishing type assault email is frequently composed as a client dissension, or official issue. This phishing trick messages are intended to take on the appearance of a email sent from a true blue business power [5]. The substance aims to be customized for higher administration and more often than not includes some distorted far-reaching concern. Whaling phishermen have additionally manufactured authority looking FBI subpoena messages and asserted that the administrator require to pop a link and introduce exceptional programming to see the subpoena.

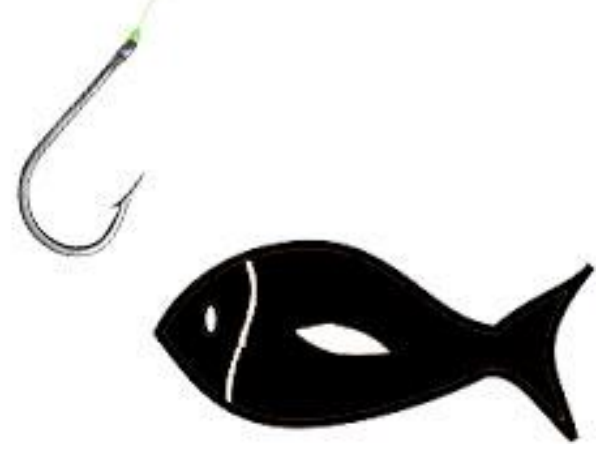

Fig 4: Whaling

\subsection{Filter Evasion}

Phishers have even begun utilizing pictures rather than fulfill to make it tougher for hostile to phishing line to distinguish message usually utilized as a part of phishing messages. Notwithstanding, this has prompted to the advancement of more modern against phishing channels that can recuperate concealed content in pictures. The attacker makes the phishing sites more undetectable by security measure such as firewalls and content filtering web proxies [8].

\section{APPROACHES TO PREVENT PHISHING ATTACK}

Place Various types of method to avoid phishing attack:

1) To Education and alert the user about various types of malpractices over the sites.

2) Frame proper techniques to punish phishing attackers.

3) Follow systematic techniques stop such practices.

4) Report and ignore such website containing malware

5) Modify the protection(security) of the website.

6) Use various types of spam filters in order to prevent the phishing mails.

\section{PREVENTION OF PHISHING}

Sites utilized for phishing identified by breaking down end client detailed information accommodation measurements. A focal procedure, for example, Google bots gets information showing secret data submitted to sites from a majority of client PCs. The got information is accumulated and broke down, for instance through measurable profiling. Through the investigation of the totaled information, irregular conduct concerning accommodation of classified data to sites is recognized, for example, a startling, fast increment in the measure of secret data submitted to a given site. Such peculiar conduct demonstrates that the site utilized for phishing. Receptive to identifying the atypical conduct, the additional move is made to shield clients from submitting classified data to that site. For instance, an alarm can sent to a proper gathering or mechanized framework, a defensive measure against the site can distributed, the site can added to a boycott or a strategy to have the site closed down can be started.

Use Dedicated Systems for Payments including solicitations and endorsement forms. Consider incapacitating email access on any framework required with installment handling. If a programmer cannot bargain the frameworks in installment 
handling, they will have a harder time acquiring installment usernames and passwords, and a much more difficult time asking for/favoring an exchange.

Use a Strong Authentication Mechanism on all installment handling frameworks. The strong authenrication mechanism would incorporate supplanting or expanding username/watchword mixes with an equipment token and PIN, or with biometrics, for example, a unique finger impression per user. An aggressor will be notable duplicate and reuse solid confirmation, for instance, a token or biometrics.

Disable the utilization of USB Flash Drives in installment preparing frameworks. In a few circles, USB streak drives frequently alluded to as "malware conveyance gadgets." Crippling USB streak drives evacuates one more potential road for the disease. Utilize instruments accessible in user's email

Be steady in utilization of anti- virus and anti- malware programming, including regular overhauls and outputs. The majority of the malware utilized as a component of a phishing assault not identified by standard anti- virus programming, but rather some of it is. Some malware pointers may not changed before an anti- virus redesign is accessible, and here and there more established variants of malware are disseminated. Moreover, the anti-infection programming can recognize optional diseases that might identified with assault.

Use reputation- based site, IP address and URL separating to guarantee that any frameworks got to from inside the organization not viewed as "awful" destinations. They can amplify this further by permitting just "white- list" get to access to locations that have particularly perceived as "great" locales, (take note of this can restrain some Internet ability).

Enforce time- of- day login and installment are preparing. Numerous fake exchanges happen after typical working hours. For example, a progression of expensive exchanges that finished at 7:00 pm Friday night may be practically disregarded until staff return and see strange exercises Monday morning.

Limit access to installment handling frameworks from cell phones, portable workstations, and frameworks situated in home workplaces. These disseminated frameworks are commonly more helpless against dangers.

Do not permit access to any interior association framework, particularly installment handling frameworks, from an claimed home PC. There is essentially no chance the association can authorize appropriate control over such a framework.

To conduct worker security mindfulness sessions. This kind of user is to train representatives on the most proficient method to distinguish phishing messages and abstain from succumbing to them. Any decrease in introduction moderates bargain and builds association's ability to recognize a heightening risk.

Explicitly impart to workers, accomplices, and customers that will never request account data through email, or send a connection to upgrade account data. Independently, there are things workers can do to abstain from turning into a casualty and trading off the uprightness of hierarchical operations:

Never open connections or connections in spontaneous messages.In general, be suspicious of all messages containing joins. If the user get an email with a link for them to click, don't click it. Explore autonomously to the goal site (for instance, by writing www.mybigbank.com into another program window) and locate the referenced area without utilizing the advantageously included connection.

Do not react to suspicious messages in any way.

Use a different PC to get to messages as opposed to using a similar PC used to start or support installments.

Report suspicious messages to administration when you get them. Phishers like to trade off sites with legal space names. These areas are harder to suspend because the space holder is additionally a casualty. Numerous site proprietors today find that they are accidentally giving offices to phishing assaults.

\section{ANTI-PHISHING TECHNIQUES}

AntiPhish depends on the start that for unpracticed, actually unsophisticated clients, it is better for software to test the trustworthiness of a webpage for the safety for its users. Dissimilar to a customer, a software will not be allow frauds or confusion traps, example, a comparable sounding area name [11]. AntiPhish is an application that coordinated into the web program that delineated in Fig 14. It monitors a client's touchy data and keeps this data safe and leads to page that is considered "genuine."or original.

When all said in done against phishing procedures can be grouped into taking after four categories [12]. Content Filtering-In this philosophy content/email are separated as it enters in the casualty's letter drop utilizing machine learning techniques, for example, Bayesian Additive Regression Trees or Support Vector Machines.

The When all said in done against phishing procedures can be grouped into taking after four categories [12]. Content Filtering-In this philosophy content/email are separated as it enters in the casualty's letter drop utilizing machine learning techniques, for example, Bayesian Additive Regression Trees or Support Vector Machines.

Boycotting Blacklist is an accumulation of known phishing Web destinations/addresses distributed by trusted substances like goggle's and Microsoft's boycott. It requires both a customer and a server segment. The client's segment actualized as either an email or program module that cooperates with a server part, which for this situation is an open Web web page that gives a rundown of known phishing destinations.

Side effect Based Prevention-Symptom-based counteractive action examinations the substance of every Web page the client visits and produces phishing cautions as indicated by the sort and number of side effects distinguished.

Area Binding-It is a customer's program based strategies where touchy data is tied to specific areas. It cautions the client when he visits an area to which client accreditation is not linked.

\section{ANALYSIS}

The most common mistake of people which leads to phishing attacks are

1. People clicking on the links in emails

2. Individuals who do not close the browser after logging out.

3. Spamming as a result of forwarding emails.

4. People who do not use Internet email security. 
5. Failing to erase hard drive when selling computer.

6. Being deceived by the common attack. Social hacking is a practice of simply asking a user to a user's login or other personal information.

\subsection{Sign's Of Phishing Attack}

1. A logo that looks distorted, stretched or shrunken.

2. The email refers you as "dear customer" rather than including your name.

3. The sign of phishing attack is due to an email that claims security threats and required action.

4. There are spelling mistakes in unknown emails. These spelling mistakes commonly used words.

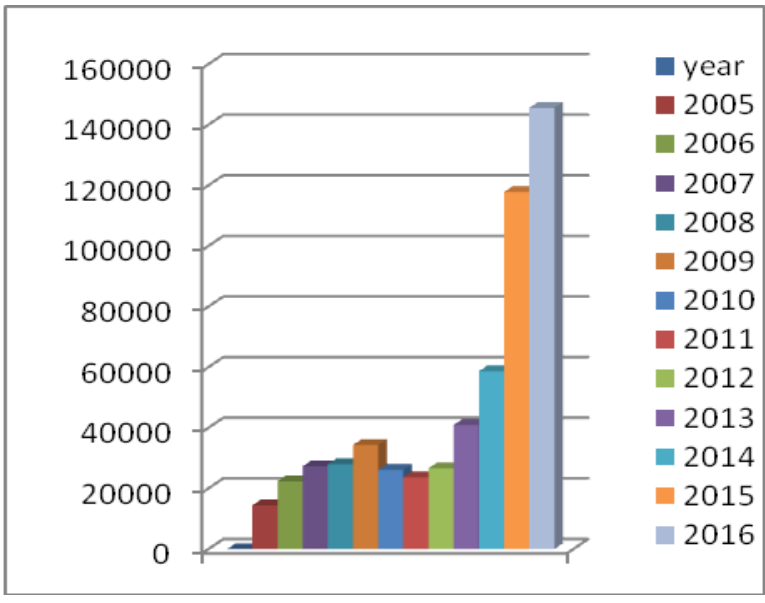

Fig 5: Statistic Of Phishing Attack

In figure 6 it is seen that the number of phishing attacks has increased drastically over the years. In 2005, the number of phishing attacks was below 20000. In 2009, the number of attacks increased to around 30000. There was 50\% increase in the number of attacks. After 2009, it is seen that there is a drop in the number of attacks for a period of 3 years after which the number of attacks increases exponentially. By 2016 the number of phishing attacks had increased to 140000 . This is an increase in the number of phishing attacks by $700 \%$.

\section{CONCLUSION}

Phishing is critical problem that result in continual threats in social media, Despite the fact that laws ordered, training is the best protection against phishing. Being somewhat suspicious of every single electronic correspondence and sites is recommended. Pay particular mind to the natural qualities feeling of earnestness, demand for confirmation, and grammar and spelling blunders. Additionally, get in the propensity for contrasting the gave URL and the free look for the organization's site,thus this paper will improve the understanding of phishing email problem and can work on developing an extension for browsers which can check the vulnerability of the network calls and alert the users if it can cause phishing attack before the actual call to the respective server.

\section{REFERENCES}

[1] S.Arun, D.Ananda, T.Selvaprabhu, B.Sivakumar, P.Revathi, and H.Shine, "Detecting Phishing Attacks In Purchasing Process Through Proactive Approach," Adv. Comput. an Int. J., vol. 3, no. 3, pp. 81-93, 2012.
[2] Q. Ma, "The process and characteristics of phishing attacks - A small international trading company case study,” J. Technol. Res., vol. 4, pp. 1-16, 2013.

[3] A. Almomani, B. B. Gupta, S. Atawneh, A. Meulenberg, and E. Almomani, "A survey of phishing email filtering techniques," IEEE Commun. Surv. Tutorials, vol. 15, no. 4, pp. 2070-2090, 2013.

[4] E. Kirda and C. Kruegel, "Protecting Users Against Phishing Attacks with AntiPhish."

[5] G. M. J, M. M. Mohideen, and S. B. N, "E-Mail Phishing - An open threat to everyone," vol. 4 , no. 2, pp. 2-5, 2014.

[6] M. N. Banu, M. A. M. C. Engineering, J. Mohamed, and C. Autonomous, "A Comprehensive Study of Phishing Attacks,” vol. 4, no. 6, pp. 783-786, 2013.

[7] U. Odaro and B. G. Sanders, "Social Engineering: Phishing for a Solution Research methodology," pp. 8896, 2007.

[8] R. Damodaram, "Study on phishing attacks and antiphishing tools," pp. 700-705, 2016.

[9] M. Chawla, "A Survey of Phishing Attack Techniques," vol. 93 , no. 3, pp. 1-4, 2014.

[10] V. Antony and D. James, "A Survey of Online Detection and Prevention of Phishing Attacks," vol. 4, no. 8, pp. 4 6, 2013.

[11] T. M. Incorporated, "Spear-Phishing Email: Most Favored APT Attack Bait," 2012.

[12] J. Chhikara and M. Rani, "International Journal of Advanced Research in Phishing \& Anti-Phishing Techniques: Case Study Phishing attacks Exploit Based," vol. 3, no. 5, pp. 458-465, 2013.

[13] M. Security, "Agenda - Phishing Intro."

[14] R. Gonzalez and M. E. Locasto, "An interdiscplinary study of phishing and spear-phishing attacks .," pp. 1-3.

[15] V. Suganya, "A Review on Phishing Attacks and Various Anti Phishing Techniques," vol. 139, no. 1, pp. 20-23, 2016.

[16] I. J. C. Network, R. Gupta, and P. K. Shukla, "Performance Analysis of Anti-Phishing Tools and Study of Classification Data Mining Algorithms for a Novel Anti-Phishing System," no. November, pp. 70-77, 2015.

[17] C. Science and M. Studies, "Development Review on Phishing: A Computer Security Threat," pp. 55-64, 2014.

[18] ]“journal8.pdf.crdownload."

[19] K. R. Sahu, "A Survey on Phishing Attacks," vol. 88, no. 10, pp. 42-45, 2014.

[20] N. Suryavanshi, M. Pradesh, A. Jain, and M. Pradesh, "A Review of Various Techniques for Detection and Prevention for Phishing Attack," pp. 41-46. 